\title{
GLOBAL SOLUTIONS OF THE EQUATION OF THE KIRCHHOFF ELASTIC ROD IN SPACE FORMS
}

\author{
SATOSHI KAWAKUBO
}

(Received 3 June 2012; accepted 18 July 2012; first published online 27 September 2012)

\begin{abstract}
The Kirchhoff elastic rod is one of the mathematical models of equilibrium configurations of thin elastic rods, and is defined to be a solution of the Euler-Lagrange equations associated to the energy with the effect of bending and twisting. In this paper, we consider Kirchhoff elastic rods in a space form. In particular, we give the existence and uniqueness of global solutions of the initial-value problem for the Euler-Lagrange equations. This implies that an arbitrary Kirchhoff elastic rod of finite length extends to that of infinite length.
\end{abstract}

2010 Mathematics subject classification: primary 49K15; secondary 34A12, 58E10, 74K10.

Keywords and phrases: Kirchhoff elastic rods, calculus of variations, ordinary differential equations, initial-value problems, global solutions.

\section{Introduction}

The elastica and the Kirchhoff elastic rod (or simply Kirchhoff rod) are both classical mathematical models of equilibrium configurations of thin elastic rods. The elastica is probably the simplest model, and is characterised as a critical curve of the energy of bending only. The Kirchhoff rod is a more complicated model, and is characterised as a critical framed curve of the energy with the effects of both bending and twisting.

Kirchhoff rods were originally considered in three-dimensional Euclidean space, but they extend naturally to an arbitrary higher-dimensional Riemannian manifold (see, for example, [18-20]). That is, we generalise the energy by replacing ordinary differentiation by covariant differentiation, and define a Kirchhoff rod as a solution of the Euler-Lagrange equations associated to the generalised energy (see [20, Section 2]).

In this paper, we consider the initial-value problem for the Euler-Lagrange equations, which are a pair of nonlinear ordinary differential equations of fourth and first orders. In particular, when the ambient space is a space form, that is, a complete

This research was partially supported by a Grant-in-Aid for Scientific Research (C) (No. 23540116) from the Japan Society for the Promotion of Science.

(c) 2012 Australian Mathematical Publishing Association Inc. 0004-9727/2012 \$16.00 
connected Riemannian manifold of constant sectional curvature, we prove that there exists a unique global solution of this initial-value problem (Theorem 2.1). Here, a global solution stands for a solution defined on the whole of $\mathbb{R}$. Since a Kirchhoff rod is, by definition, arclength-parametrised, this result implies that a Kirchhoff rod of finite length extends to that of infinite length.

We note that in general, solution curves of a variational problem do not necessarily extend to global solutions. For instance, there exist biminimal curves in the Euclidean plane [33] or in a general two-dimensional space form [14] whose curvatures blow up in finite time. Here, a unit-speed curve is called biminimal if it is a critical point of the bienergy functional for normal variations $[14,33]$. These results imply that there exist biminimal curves which cannot extend to global solutions.

Since the time of Euler in the 1730s, many authors have been studying various models of thin elastic rods. The Kirchhoff rod is the simplest model in which bending and twisting both play a role. In the case where the ambient space is the Euclidean space $\mathbb{R}^{n}$, especially $\mathbb{R}^{2}$ or $\mathbb{R}^{3}$, there is a rich literature on more general models of thin elastic rods. To give one example, Antman [1] proved existence and regularity theorems for solutions to boundary-value problems for a very general class of energies and boundary conditions; for more results, see [2, 34] and references therein. Also, various researchers have been extensively studying Kirchhoff rods in $\mathbb{R}^{3}$. For instance, it is known that by taking cylindrical coordinates, the centrelines of all Kirchhoff rods are explicitly expressed by Jacobi sn functions and elliptic integrals [31, 38, 39]; see also [3, 11, 15, 24, 35], for example.

Meanwhile, elasticae or their generalisations in Riemannian manifolds, except Euclidean space, have been investigated since the 1980s (see, for example, $[4-7,9,12,13,16,26-29,32,36,37])$. To give an example of the results on global solutions, Popiel and Noakes [37, Theorem 3.1] proved that when the ambient space is a compact Lie group with a bi-invariant Riemannian metric, there exists a unique global solution of the initial-value problem for the equation of the elastica.

Kirchhoff rods in three-dimensional simply connected space forms $\mathbb{R}^{3}, S^{3}, H^{3}$ are investigated in $[17,18,20,21,30]$, for example. It is known that the Frenet curvature and torsion of the centrelines of all Kirchhoff rods in $\mathbb{R}^{3}[31,38,39]$ or in $S^{3}, H^{3}$ [20] are explicitly expressed in terms of Jacobi sn function. By using these explicit formulas, it follows immediately that a Kirchhoff rod of finite length in $\mathbb{R}^{3}, S^{3}, H^{3}$ extends to that of infinite length. Moreover, even in the case of $S^{3}, H^{3}$, it is known that by taking similar coordinates to the cylindrical coordinates in $\mathbb{R}^{3}$, the centrelines themselves of all Kirchhoff rods are expressed explicitly by Jacobi sn functions and elliptic integrals [21].

On the other hand, there are not so many studies on Kirchhoff rods in space forms of dimension $n \geq 4$. In [23], the present author constructed examples of Kirchhoff rods whose centrelines are fully immersed in $\mathbb{R}^{n}, S^{n}, H^{n}(n \geq 4)$. In the case of $n \geq 4$, however, explicit formulas of the higher-order Frenet curvatures, or other kinds of curvatures of all Kirchhoff rods are not known. Thus, unlike the case of $n=3$, it is not 
clear if an arbitrary Kirchhoff rod of finite length extends to that of infinite length. In this paper, we answer this question in the affirmative.

The outline of the proof of the main theorem (Theorem 2.1) is as follows. The point is to use the natural curvatures of an arclength-parametrised curve, which are different from the ordinary Frenet curvatures (see, for example, [8, 31]). We reduce the Euler-Lagrange equations to the equation for natural curvature vector, which is an $\mathbb{R}^{n-1}$-valued second-order ordinary differential equation. Then we construct a first integral of the equation, and derive an estimate for solutions of the equation. By using this estimate, we show the existence of a global solution of the initial-value problem for the Euler-Lagrange equations.

The rest of the paper is organised as follows. In Section 2 we define a Kirchhoff rod, formulate the initial-value problem for the Euler-Lagrange equations and give the precise statement of our main theorem (Theorem 2.1). In Section 3 we recall the notions and properties of the natural frame and natural curvatures of an arclengthparametrised curve. In Section 4 we construct a first integral of the equation for natural curvature vector (Proposition 4.1). In Section 5 we derive an estimate for solutions of the equation (Lemma 5.1), and prove the main theorem.

This paper is the complete version of the announcement [22] in Japanese.

\section{Preliminaries and results}

In this section, we first define a Kirchhoff rod in a Riemannian manifold. Then we formulate the initial-value problem for the Euler-Lagrange equations, and give the precise statement of our main theorem (Theorem 2.1).

Unless otherwise specified, all manifolds, curves, vector fields etc. are assumed to be of class $C^{\infty}$. Let $\mathscr{M}$ be an $n$-dimensional $(n \geq 2)$ complete connected Riemannian manifold with metric $\langle *, *\rangle$. We denote by $T \mathscr{M}$ the tangent bundle of $\mathscr{M}$, by $\nabla$ the Levi-Civita connection in $T \mathscr{M}$ and by $R$ the Riemannian curvature tensor. Here, we use the sign convention of $R$ corresponding to that of [25], that is, $R(X, Y) Z=$ $\nabla_{X} \nabla_{Y} Z-\nabla_{Y} \nabla_{X} Z-\nabla_{[X, Y]} Z$.

Let $\gamma=\gamma(t):\left[t_{1}, t_{2}\right] \rightarrow \mathscr{M}$ be a unit-speed, that is, arclength-parametrised curve in $\mathscr{M}$, and $T(t)=\gamma^{\prime}(t)$ the tangent vector to $\gamma$. The twisting of an elastic rod cannot be represented by a curve $\gamma$ only. (Note that the Frenet torsion $\tau$ or the higherorder Frenet curvatures of $\gamma$ are not directly related to the twisting of the elastic rod.) To describe how the elastic rod is twisted, we utilise an orthonormal frame field $M=\left(M_{1}, M_{2}, \ldots, M_{n-1}\right)$ in the normal bundle $T^{\perp} \mathscr{M}$ along $\gamma$. We call such a pair $\{\gamma, M\}$ a unit-speed curve with adapted orthonormal frame, and $\gamma$ the centreline of $\{\gamma, M\}$.

Now, let $v$ be a fixed positive constant, which is determined by the material of a given rod. We define the energy $\mathfrak{I}$ as follows:

$$
\mathfrak{I}(\{\gamma, M\})=\int_{t_{1}}^{t_{2}}\left|\nabla_{T} T\right|^{2} d t+v \sum_{i=1}^{n-1} \int_{t_{1}}^{t_{2}}\left|\nabla_{T}^{\perp} M_{i}\right|^{2} d t
$$


where $\nabla^{\perp}$ denotes the normal connection in $T^{\perp} \mathscr{M}$, so that $\nabla_{T}^{\perp} M_{i}=\nabla_{T} M_{i}-$ $\left\langle\nabla_{T} M_{i}, T\right\rangle T$. The first term of $\mathfrak{I}(\{\gamma, M\})$ expresses the energy of bending, and the second term that of twisting. We call $\{\gamma, M\}$ a Kirchhoff $\operatorname{rod}$ if $\{\gamma, M\}$ is a critical point of $\mathfrak{I}$ with respect to the variations of unit-speed curves with adapted orthonormal frames which preserve the end points $\gamma\left(t_{1}\right), \gamma\left(t_{2}\right)$ and the orthonormal frames $\left(T\left(t_{1}\right), M\left(t_{1}\right)\right),\left(T\left(t_{2}\right), M\left(t_{2}\right)\right)$ at the end points. More precisely, a Kirchhoff rod is defined to be a solution of the associated Euler-Lagrange equations:

$$
\begin{gathered}
\nabla_{T}\left(2\left(\nabla_{T}\right)^{2} T+\left(3\left|\nabla_{T} T\right|^{2}-\mu+v|a|^{2}\right) T\right)-4 v \sum_{i, j=1}^{n-1}\left\langle\left(\nabla_{T}\right)^{2} T, M_{i}\right\rangle a_{j i} M_{j} \\
+2 R\left(\nabla_{T} T, T\right) T+2 v \sum_{i, j=1}^{n-1} a_{j i} R\left(M_{j}, M_{i}\right) T=0, \\
\left(\nabla_{T}^{\perp} M_{1}, \ldots, \nabla_{T}^{\perp} M_{n-1}\right)=\left(M_{1}, \ldots, M_{n-1}\right) a,
\end{gathered}
$$

where $\mu \in \mathbb{R}$ and $a=\left(a_{j i}\right)_{1 \leq j, i \leq n-1} \in \mathfrak{D}(n-1)$. Here, $\mathfrak{o}(n-1)$ stands for the Lie algebra of all skew-symmetric matrices of size $n-1$. Also, $|a|$ stands for the Euclidean norm of the $(n-1) \times(n-1)$ matrix $a$. The system of (2.1) and (2.2) is equivalent to the system of (2.1) and (2.2) in [20]. For the derivation of these equations, see [20, Section 2].

Definition. Let $\{\gamma, M\}$ be a unit-speed curve with adapted orthonormal frame in $\mathscr{M}$. We call $\{\gamma, M\}$ a Kirchhoff rod if there exist $\mu \in \mathbb{R}$ and $a \in \mathfrak{D}(n-1)$ such that (2.1) and (2.2) hold. The matrix $a$ is uniquely determined, and is called the twist matrix of $\{\gamma, M\}$.

We note that the matrix $a$ in (2.2) is independent of $t$. Therefore, we see that if $\{\gamma, M\}$ is a Kirchhoff rod, then the integrand of the second term of $\mathfrak{I}(\{\gamma, M\})$ is independent of $t$. Physically, this means that the twist of an elastic rod in equilibrium is uniformly distributed along the centreline.

We formulate the initial-value problem for the Euler-Lagrange equations (2.1) and (2.2). Suppose that $\{\gamma, M\}$ is a Kirchhoff rod in $\mathscr{M}$. Since $\gamma$ is unit-speed, the three vectors $T, \nabla_{T} T$ and $\left(\nabla_{T}\right)^{2} T$ along $\gamma$ must satisfy some conditions. (The argument is similar to the case of elasticae; see [37].) First, $|T|=1$ holds. Next, differentiating $|T|^{2}=1$ yields $\left\langle\nabla_{T} T, T\right\rangle=0$. Differentiating this identity further, it follows that $\left\langle\left(\nabla_{T}\right)^{2} T, T\right\rangle=-\left|\nabla_{T} T\right|^{2}$. Thus, the initial-value problem is formulated as follows.

Problem. Let $t_{0}, \mu \in \mathbb{R}, a \in \mathfrak{p}(n-1)$ and $x \in \mathscr{M}$. Suppose that $V_{1}, V_{2}, V_{3} \in T_{x} \mathscr{M}$ and $L_{1}, \ldots, L_{n-1} \in T_{x} \mathscr{M}$ are such that

$$
\begin{aligned}
& \left|V_{1}\right|=1, \quad\left\langle V_{2}, V_{1}\right\rangle=0, \quad\left\langle V_{3}, V_{1}\right\rangle=-\left|V_{2}\right|^{2}, \\
& \left\langle L_{i}, V_{1}\right\rangle=0 \quad(1 \leq i \leq n-1), \quad\left\langle L_{i}, L_{j}\right\rangle=\delta_{i j} \quad(1 \leq i, j \leq n-1) .
\end{aligned}
$$


Find a unit-speed curve with adapted orthonormal frame $\{\gamma(t), M(t)\}$ in $\mathscr{M}$ defined on an interval containing $t_{0}$ which satisfies (2.1), (2.2) and the following initial conditions:

$$
\begin{aligned}
& \gamma\left(t_{0}\right)=x, \quad T\left(t_{0}\right)=V_{1},\left.\quad \nabla_{T} T\right|_{t=t_{0}}=V_{2},\left.\quad\left(\nabla_{T}\right)^{2} T\right|_{t=t_{0}}=V_{3}, \\
& \left.M\right|_{t=t_{0}}=\left.\left(M_{1}, \ldots, M_{n-1}\right)\right|_{t=t_{0}}=\left(L_{1}, \ldots, L_{n-1}\right) .
\end{aligned}
$$

In the case where $\mathscr{M}$ is a space form, we prove that there exists a unique global solution of this initial-value problem. To be precise, the following main theorem holds.

Theorem 2.1. Suppose that $\mathscr{M}$ is a space form. Let $t_{0}, \mu \in \mathbb{R}, a \in \mathfrak{v}(n-1), x \in \mathscr{M}$ and let $V_{1}, V_{2}, V_{3}, L_{1}, \ldots, L_{n-1} \in T_{x} \mathscr{M}$ satisfy condition (2.3). Then there exists a unique unit-speed curve with adapted orthonormal frame $\{\gamma, M\}$ in $\mathscr{M}$ defined on the whole of $\mathbb{R}$ satisfying (2.1), (2.2) and initial conditions (2.4).

\section{Natural frames and curvatures}

In this section, we recall the definitions and some properties of the natural frame and natural curvatures of a unit-speed curve (see, for example, [8, 31]).

Let $\mathscr{M}$ be an $n$-dimensional $(n \geq 2)$ complete Riemannian manifold, and let $I=\left(t_{1}, t_{2}\right)$, where $-\infty \leq t_{1}<t_{2} \leq \infty$. Let $\gamma: I \rightarrow \mathscr{M}$ be a unit-speed curve. An orthonormal frame field $\left(T, P_{1}, \ldots, P_{n-1}\right)$ along $\gamma$ is said to be a natural frame along $\gamma$ if $\nabla_{T}^{\perp} P_{i}=0$ holds on $I$ for $i=1, \ldots, n-1$. For a given $\gamma$, there exists a natural frame along $\gamma$. Indeed, we take an orthonormal frame $P^{0}=\left(P_{1}^{0}, \ldots, P_{n-1}^{0}\right)$ of the normal vector space at a point $\gamma\left(t_{0}\right)$ on the curve $\gamma$, and let $P_{i}=P_{i}(t), i=1, \ldots, n-1$, be the normal vector field along $\gamma$ obtained by parallel translating $P_{i}^{0}$ along $\gamma$ with respect to the normal connection $\nabla^{\perp}$. Then $\left(T, P_{1}, \ldots, P_{n-1}\right)$ is a natural frame along $\gamma$.

For a natural frame $\left(T, P_{1}, \ldots, P_{n-1}\right)$ along $\gamma$, we set $k_{i}=\left\langle\nabla_{T} T, P_{i}\right\rangle, \quad i=$ $1, \ldots, n-1$, and $\mathbf{k}={ }^{t}\left(k_{1}, \ldots, k_{n-1}\right)$. Then it follows that

$$
\left(\nabla_{T} T, \nabla_{T} P_{1}, \ldots, \nabla_{T} P_{n-1}\right)=\left(T, P_{1}, \ldots, P_{n-1}\right)\left(\begin{array}{cc}
0 & -{ }^{t} \mathbf{k} \\
\mathbf{k} & 0
\end{array}\right) .
$$

These functions $k_{1}, \ldots, k_{n-1}$ are said to be the natural curvatures of $\gamma$ with respect to the natural frame $\left(T, P_{1}, \ldots, P_{n-1}\right)$. Also, the map $\mathbf{k}: I \rightarrow \mathbb{R}^{n-1}$ is said to be the natural curvature vector of $\gamma$ with respect to the natural frame $\left(T, P_{1}, \ldots, P_{n-1}\right)$.

For a given unit-speed curve $\gamma$, the natural frame and the natural curvature vector are determined only up to an action by the orthogonal group $O(n-1)$. To be precise, if $\left(T, P_{1}, \ldots, P_{n-1}\right)$ is a natural frame along $\gamma$, then the totality of natural frames along $\gamma$ consists of frames of the form $\left(T,\left(P_{1}, \ldots, P_{n-1}\right) \varphi\right)$, where $\varphi \in O(n-1)$. Also, the natural curvature vector of $\gamma$ with respect to the natural frame $\left(T,\left(P_{1}, \ldots, P_{n-1}\right) \varphi\right)$ becomes $\varphi^{-1} \mathbf{k}$.

Compared to the Frenet frame, the natural frame has the advantage that it can be defined even on a point where $\nabla_{T} T=0$.

In the same way as in the case of the Frenet frame, the following proposition holds, whose proof is omitted. Two curves $\gamma_{1}, \gamma_{2}: I \rightarrow \mathscr{M}$ are called congruent if there exists an isometry $\Phi$ of $\mathscr{M}$ such that $\gamma_{2}=\Phi \circ \gamma_{1}$. 
Proposition 3.1. The following statements hold.

(i) For any $\mathbf{k}: I \rightarrow \mathbb{R}^{n-1}$, there exists a unit-speed curve $\gamma: I \rightarrow \mathscr{M}$ such that the natural curvature vector of $\gamma$ with respect to a natural frame corresponds to $\mathbf{k}$. In more detail, the following holds. Let $\mathbf{k}: I \rightarrow \mathbb{R}^{n-1}$ be any map. Let $t_{0} \in I$ and let $\left(T^{0}, P_{1}^{0}, \ldots, P_{n-1}^{0}\right)$ be an orthonormal frame of the tangent space $T_{x} \mathscr{M}$ at a point $x \in \mathscr{M}$. Then there exists a unique unit-speed curve with adapted orthonormal frame $\left\{\gamma,\left(P_{1}, \ldots, P_{n-1}\right)\right\}$ satisfying the following: $\left(\gamma^{\prime}, P_{1}, \ldots, P_{n-1}\right)$ is a natural frame along $\gamma$, and the natural curvature vector of $\gamma$ with respect to $\left(\gamma^{\prime}, P_{1}, \ldots, P_{n-1}\right)$ corresponds to $\mathbf{k}$, and $\gamma\left(t_{0}\right)=x$ and $\left(\gamma^{\prime}\left(t_{0}\right), P_{1}\left(t_{0}\right), \ldots, P_{n-1}\left(t_{0}\right)\right)=\left(T^{0}, P_{1}^{0}, \ldots, P_{n-1}^{0}\right)$.

(ii) Suppose that $\mathscr{M}=\mathbb{R}^{n}, S^{n}, H^{n}$ of constant sectional curvature. Let $\gamma, \widetilde{\gamma}: I \rightarrow \mathscr{M}$ be two unit-speed curves. We denote by $\mathbf{k}$ (respectively, $\widetilde{\mathbf{k}}$ ) the natural curvature vector of $\gamma$ (respectively, $\widetilde{\gamma})$ with respect to a natural frame. Then $\gamma$ and $\widetilde{\gamma}$ are congruent if and only if $\widetilde{\mathbf{k}}=\varphi \mathbf{k}$ holds for some $\varphi \in O(n-1)$.

\section{First integrals}

In this section, we first reduce the Euler-Lagrange equations (2.1) and (2.2) in a space form to the equation for natural curvature vector, and construct a first integral of this equation.

Throughout the rest of the paper, we assume that $\mathscr{M}$ is a space form. We denote by $G \in \mathbb{R}$ the constant sectional curvature. Then (2.1) and (2.2) are reduced to

$$
\begin{aligned}
& \nabla_{T}\left(2\left(\nabla_{T}\right)^{2} T+\left(3\left|\nabla_{T} T\right|^{2}-\mu+2 G+v|a|^{2}\right) T\right) \\
& \quad-4 v \sum_{i, j=1}^{n-1}\left\langle\left(\nabla_{T}\right)^{2} T, M_{i}\right\rangle a_{j i} M_{j}=0, \\
& \left(\nabla_{T}^{\perp} M_{1}, \ldots, \nabla_{T}^{\perp} M_{n-1}\right)=\left(M_{1}, \ldots, M_{n-1}\right) a .
\end{aligned}
$$

We seek the condition for a unit-speed curve with adapted orthonormal frame to satisfy (4.1) and (4.2) in terms of the natural frame and natural curvature vector.

Let $\{\gamma, M\}$ be a unit-speed curve with adapted orthonormal frame in $\mathscr{M}$ defined on $I=\left(t_{1}, t_{2}\right)$, where $-\infty \leq t_{1}<t_{2} \leq \infty$. Let $\left(T, P_{1}, \ldots, P_{n-1}\right)$ be a natural frame along $\gamma$, and $\mathbf{k}: I \rightarrow \mathbb{R}^{n-1}$ the natural curvature vector of $\gamma$ with respect to the natural frame $\left(T, P_{1}, \ldots, P_{n-1}\right)$. We fix a point $t_{0} \in I$. Then there exists a unique $\varphi \in O(n-1)$ such that

$$
\left(M_{1}\left(t_{0}\right), \ldots, M_{n-1}\left(t_{0}\right)\right)=\left(P_{1}\left(t_{0}\right), \ldots, P_{n-1}\left(t_{0}\right)\right) \varphi .
$$

Let $\mu \in \mathbb{R}$ and $a \in \mathfrak{D}(n-1)$. We show that $\{\gamma, M\}$ satisfies (4.1) and (4.2) if and only if the following two equations hold:

$$
\begin{aligned}
& 2 \mathbf{k}^{\prime \prime}-4 v \varphi a \varphi^{-1} \mathbf{k}^{\prime}+\left(|\mathbf{k}|^{2}-\mu+2 G+v|a|^{2}\right) \mathbf{k}=\mathbf{0}, \\
& \left(M_{1}, \ldots, M_{n-1}\right)=\left(P_{1}, \ldots, P_{n-1}\right) \varphi \exp \left(\left(t-t_{0}\right) a\right) .
\end{aligned}
$$


Suppose that (4.1) and (4.2) hold. Since $\left(M_{1}, \ldots, M_{n-1}\right)$ is expressed as

$$
\left(M_{1}, \ldots, M_{n-1}\right)=\left(P_{1}, \ldots, P_{n-1}\right) \xi
$$

for some map $\xi: I \rightarrow O(n-1)$, it follows that

$$
\left(\nabla_{T}^{\perp} M_{1}, \ldots, \nabla_{T}^{\perp} M_{n-1}\right)=\left(M_{1}, \ldots, M_{n-1}\right) \xi^{-1} \xi^{\prime} .
$$

Comparing this expression with (4.2) yields $a=\xi^{-1} \xi^{\prime}$. Thus, by $\xi\left(t_{0}\right)=\varphi$,

$$
\xi(t)=\varphi \exp \left(\left(t-t_{0}\right) a\right)
$$

and hence (4.4) holds.

Next, it follows from (3.1) that

$$
\begin{aligned}
& \nabla_{T} T=\left(P_{1}, \ldots, P_{n-1}\right) \mathbf{k}, \quad\left(\nabla_{T}\right)^{2} T=-|\mathbf{k}|^{2} T+\left(P_{1}, \ldots, P_{n-1}\right) \mathbf{k}^{\prime}, \\
& \left(\nabla_{T}\right)^{3} T=-3\left\langle\mathbf{k}^{\prime}, \mathbf{k}\right\rangle T+\left(P_{1}, \ldots, P_{n-1}\right)\left(\mathbf{k}^{\prime \prime}-|\mathbf{k}|^{2} \mathbf{k}\right),
\end{aligned}
$$

where $\langle *, *\rangle$ (respectively, $|*|)$ stands for the Euclidean inner product (respectively, norm) of $\mathbb{R}^{n-1}$. Also,

$$
\begin{aligned}
\sum_{i, j=1}^{n-1}\left\langle\left(\nabla_{T}\right)^{2} T, M_{i}\right\rangle a_{j i} M_{j} & =\sum_{i, j=1}^{n-1}\left\langle\left(M_{1}, \ldots, M_{n-1}\right) \xi^{-1} \mathbf{k}^{\prime}, M_{i}\right\rangle a_{j i} M_{j} \\
& =\left(M_{1}, \ldots, M_{n-1}\right) a \xi^{-1} \mathbf{k}^{\prime}=\left(P_{1}, \ldots, P_{n-1}\right) \varphi a \varphi^{-1} \mathbf{k}^{\prime} .
\end{aligned}
$$

Therefore, the left-hand side of (4.1) is written as

$$
\left(P_{1}, \ldots, P_{n-1}\right)\left(2 \mathbf{k}^{\prime \prime}-4 v \varphi a \varphi^{-1} \mathbf{k}^{\prime}+\left(|\mathbf{k}|^{2}-\mu+2 G+v|a|^{2}\right) \mathbf{k}\right),
$$

and hence (4.3) holds.

Conversely, it is easily verified that if (4.3) and (4.4) hold, then $\{\gamma, M\}$ satisfies (4.1) and (4.2).

We now construct a first integral of (4.3).

Proposition 4.1. Let $\mu \in \mathbb{R}, a \in \mathfrak{p}(n-1), \varphi \in O(n-1)$ and $I=\left(t_{1}, t_{2}\right)$, where $-\infty \leq t_{1}<$ $t_{2} \leq \infty$. If $\mathbf{k}: I \rightarrow \mathbb{R}^{n-1}$ is a solution of (4.3), then

$$
\left|\mathbf{k}^{\prime}\right|^{2}+\frac{1}{4}|\mathbf{k}|^{4}+\frac{1}{2}\left(-\mu+2 G+v|a|^{2}\right)|\mathbf{k}|^{2}
$$

is constant on $I$.

Proof. Taking the inner product of (4.3) with $\mathbf{k}^{\prime}$ yields

$$
2\left\langle\mathbf{k}^{\prime \prime}, \mathbf{k}^{\prime}\right\rangle-4 v\left\langle\varphi a \varphi^{-1} \mathbf{k}^{\prime}, \mathbf{k}^{\prime}\right\rangle+\left(|\mathbf{k}|^{2}-\mu+2 G+v|a|^{2}\right)\left\langle\mathbf{k}, \mathbf{k}^{\prime}\right\rangle=0 .
$$

Since $\varphi a \varphi^{-1}$ is skew-symmetric, the second term of the left-hand side vanishes. Also, by $2\left\langle\mathbf{k}^{\prime \prime}, \mathbf{k}^{\prime}\right\rangle=\left(\left|\mathbf{k}^{\prime}\right|^{2}\right)^{\prime}$ and $\left\langle\mathbf{k}^{\prime}, \mathbf{k}\right\rangle=\left(|\mathbf{k}|^{2}\right)^{\prime} / 2$, it follows that

$$
\left(\left|\mathbf{k}^{\prime}\right|^{2}\right)^{\prime}+\frac{1}{2}\left(|\mathbf{k}|^{2}-\mu+2 G+v|a|^{2}\right)\left(|\mathbf{k}|^{2}\right)^{\prime}=0 .
$$

Hence

$$
\left(\left|\mathbf{k}^{\prime}\right|^{2}+\frac{1}{4}|\mathbf{k}|^{4}+\frac{1}{2}\left(-\mu+2 G+v|a|^{2}\right)|\mathbf{k}|^{2}\right)^{\prime}=0,
$$

which completes the proof. 


\section{Proof of main theorem}

In this section, by using the first integral obtained in Proposition 4.1, we derive an estimate for solutions of (4.3) (Lemma 5.1), and prove the main theorem.

Lemma 5.1. Suppose that $\mu \in \mathbb{R}, a \in \mathfrak{p}(n-1), \varphi \in O(n-1)$ and $I=\left(t_{1}, t_{2}\right)$, where $-\infty \leq t_{1}<t_{2} \leq \infty$. If $\mathbf{k}: I \rightarrow \mathbb{R}^{n-1}$ is a solution of (4.3), then there exists a positive constant $C$ independent of $t$ such that $|\mathbf{k}(t)|^{2}+\left|\mathbf{k}^{\prime}(t)\right|^{2} \leq C$ for all $t \in I$.

Proof. By Proposition 4.1, there exists $C_{1} \in \mathbb{R}$ such that

$$
\left|\mathbf{k}^{\prime}\right|^{2}+\frac{1}{4}|\mathbf{k}|^{4}+\frac{1}{2}\left(-\mu+2 G+v|a|^{2}\right)|\mathbf{k}|^{2}=C_{1}
$$

for all $t \in I$. Thus,

$$
\left|\mathbf{k}^{\prime}\right|^{2}=Q\left(|\mathbf{k}|^{2}\right)
$$

where

$$
Q(y)=-\frac{1}{4} y^{2}-\frac{1}{2}\left(-\mu+2 G+v|a|^{2}\right) y+C_{1} .
$$

Since the quadratic coefficient of $Q(y)$ is negative, there exists a positive constant $C_{2}$ such that $Q(y) \leq C_{2}$ for all $y \in \mathbb{R}$. Therefore, by (5.1), $\left|\mathbf{k}^{\prime}\right|^{2} \leq C_{2}$ for all $t \in I$.

Also, since the set of all $y \in \mathbb{R}$ such that $Q(y) \geq 0$ is bounded, it follows from (5.1) that $|\mathbf{k}(t)|^{2}$ is bounded on $I$. That is, there exists a positive constant $C_{3}$ such that $|\mathbf{k}(t)|^{2} \leq C_{3}$ for all $t \in I$. Hence $|\mathbf{k}(t)|^{2}+\left|\mathbf{k}^{\prime}(t)\right|^{2} \leq C_{2}+C_{3}$ for all $t \in I$, which completes the proof of the lemma.

We now give the proof of the main theorem.

Proof of Theorem 2.1. We reduce the initial-value problem (4.1), (4.2) and (2.4) for $\{\gamma, M\}$ to that for $\mathbf{k}$.

By (2.3), there exist unique vectors $\mathbf{q}, \mathbf{p} \in \mathbb{R}^{n-1}$ such that

$$
V_{2}=\left(L_{1}, \ldots, L_{n-1}\right) \mathbf{q}, \quad V_{3}=-\left|V_{2}\right|^{2} V_{1}+\left(L_{1}, \ldots, L_{n-1}\right) \mathbf{p} .
$$

We consider the following initial-value problem for $\mathbf{k}$ : find a solution $\mathbf{k}(t)=$ ${ }^{t}\left(k_{1}(t), \ldots, k_{n-1}(t)\right)$ of the equation

$$
2 \mathbf{k}^{\prime \prime}-4 v a \mathbf{k}^{\prime}+\left(|\mathbf{k}|^{2}-\mu+2 G+v|a|^{2}\right) \mathbf{k}=\mathbf{0}
$$

satisfying the initial conditions

$$
\mathbf{k}\left(t_{0}\right)=\mathbf{q}, \quad \mathbf{k}^{\prime}\left(t_{0}\right)=\mathbf{p} .
$$

For this problem, the following claim holds.

Claim. There exists a unique global solution $\mathbf{k}: \mathbb{R} \rightarrow \mathbb{R}^{n-1}$ of the initial-value problem (5.3) and (5.4). 
Assuming this claim for the moment, we complete the proof. Using the $\mathbf{k}$ of this claim, we define $\{\gamma, M\}$ on the whole of $\mathbb{R}$ as follows. By Proposition 3.1(i), there exists a unique unit-speed curve with adapted orthonormal frame $\left\{\gamma,\left(P_{1}, \ldots, P_{n-1}\right)\right\}$ defined on $\mathbb{R}$ satisfying the following: $\left(T\left(=\gamma^{\prime}\right), P_{1}, \ldots, P_{n-1}\right)$ is a natural frame along $\gamma$ and the natural curvature vector of $\gamma$ with respect to $\left(T, P_{1}, \ldots, P_{n-1}\right)$ corresponds to $\mathbf{k}$, and

$$
\gamma\left(t_{0}\right)=x,\left.\quad\left(T, P_{1}, \ldots, P_{n-1}\right)\right|_{t=t_{0}}=\left(V_{1}, L_{1}, \ldots, L_{n-1}\right) .
$$

Also, we set $\left(M_{1}, \ldots, M_{n-1}\right)=\left(P_{1}, \ldots, P_{n-1}\right) \exp \left(\left(t-t_{0}\right) a\right)$. Then, since (4.3) and (4.4) with $\varphi=e$ hold, this $\{\gamma, M\}$ satisfies (4.1) and (4.2). Here, $e$ stands for the identity matrix of size $n-1$. Also, it follows from (5.2), (5.4) and the first and second identities of (4.5) that $\{\gamma, M\}$ satisfies the initial conditions (2.4). Hence the proof of the existence part of Theorem 2.1 is complete.

Next we show the uniqueness. Suppose that a unit-speed curve with adapted orthonormal frame $\{\widetilde{\gamma}, \widetilde{M}\}$ defined on $\mathbb{R}$ also satisfies (4.1), (4.2) and (2.4). Set $\widetilde{T}=\widetilde{\gamma}^{\prime}$ and let $\left(\widetilde{T}, \widetilde{P}_{1}, \ldots, \widetilde{P}_{n-1}\right)$ be the natural frame along $\widetilde{\gamma}$ satisfying $\left.\left(\widetilde{T}, \widetilde{P}_{1}, \ldots, \widetilde{P}_{n-1}\right)\right|_{t=t_{0}}=\left(V_{1}, L_{1}, \ldots, L_{n-1}\right)$, and let $\widetilde{\mathbf{k}}$ be the natural curvature vector of $\widetilde{\gamma}$ with respect to $\left(\widetilde{T}, \widetilde{P}_{1}, \ldots, \widetilde{P}_{n-1}\right)$. Since $\{\widetilde{\gamma}, \widetilde{M}\}$ is a solution of the initialvalue problem (4.1), (4.2) and (2.4), $\widetilde{\mathbf{k}}: \mathbb{R} \rightarrow \mathbb{R}^{n-1}$ is a solution of the initialvalue problem (5.3) and (5.4). Thus, by the uniqueness part of the above claim, $\mathbf{k}=\widetilde{\mathbf{k}}$ holds. Therefore, it follows from the uniqueness part of Proposition 3.1(i) that $\left\{\gamma,\left(P_{1}, \ldots, P_{n-1}\right)\right\}=\left\{\widetilde{\gamma},\left(\widetilde{P}_{1}, \ldots, \widetilde{P}_{n-1}\right)\right\}$. So, $M=\left(P_{1}, \ldots, P_{n-1}\right) \exp \left(\left(t-t_{0}\right) a\right)=$ $\left(\widetilde{P}_{1}, \ldots, \widetilde{P}_{n-1}\right) \exp \left(\left(t-t_{0}\right) a\right)=\widetilde{M}$. Hence $\{\gamma, M\}=\{\widetilde{\gamma}, \widetilde{M}\}$, which completes the proof of Theorem 2.1.

We prove the claim. It is sufficient to prove that there exists a unique global solution $\left(\frac{\mathbf{k}}{\mathbf{k}}\right)={ }^{t}\left(k_{1}, \ldots, k_{n-1}, \widehat{k}_{1}, \ldots, \widehat{k}_{n-1}\right): \mathbb{R} \rightarrow \mathbb{R}^{2(n-1)}$ of the following initial-value problem:

$$
\begin{gathered}
\frac{d}{d t}\left(\begin{array}{l}
\mathbf{k} \\
\mathbf{k}
\end{array}\right)=\left(\begin{array}{c}
\widehat{\mathbf{k}} \\
2 v a \widehat{\mathbf{k}}-\frac{1}{2}\left(|\mathbf{k}|^{2}-\mu+2 G+v|a|^{2}\right) \mathbf{k}
\end{array}\right), \\
\left(\begin{array}{l}
\mathbf{k}\left(t_{0}\right) \\
\widehat{\mathbf{k}}\left(t_{0}\right)
\end{array}\right)=\left(\begin{array}{l}
\mathbf{q} \\
\mathbf{p}
\end{array}\right) .
\end{gathered}
$$

The right-hand side of (5.5) satisfies a Lipschitz condition with respect to ${ }^{t}(\mathbf{k}, \widehat{\mathbf{k}})$ on any compact set of $\mathbb{R}^{2(n-1)}$. Thus, by an argument similar to that in $[10$, Section 2 of Ch. 1], we see that a solution ${ }^{t}(\mathbf{k}, \widehat{\mathbf{k}}): \mathbb{R} \rightarrow \mathbb{R}^{2(n-1)}$ of the initial-value problem (5.5), (5.6) is unique.

Next we show the existence of a global solution of the initial-value problem (5.5), (5.6). It follows from the vector equation version of the Picard-Lindelöf theorem [10, Theorem 3.1 of Ch. 1] that there exist $\varepsilon>0$ and a unique solution ${ }^{t}(\mathbf{k}, \widehat{\mathbf{k}}):\left(t_{0}-\varepsilon, t_{0}+\varepsilon\right) \rightarrow \mathbb{R}^{2(n-1)}$ of (5.5) satisfying the initial condition (5.6).

We show that this local solution ${ }^{t}(\mathbf{k}, \widehat{\mathbf{k}})$ on $\left(t_{0}-\varepsilon, t_{0}+\varepsilon\right)$ can be continued to a solution on $\left(t_{0}-\varepsilon, \infty\right)$. In order to obtain a contradiction, we suppose that ${ }^{t}(\mathbf{k}, \widehat{\mathbf{k}})$ 
cannot be continued to a solution on $\left(t_{0}-\varepsilon, \infty\right)$. Then there exists a real number $t_{m}\left(\geq t_{0}+\varepsilon\right)$ satisfying the following: ${ }^{t}(\mathbf{k}, \widehat{\mathbf{k}})$ has a continuation to $\left(t_{0}-\varepsilon, t_{m}\right)$, but cannot be continued beyond $t_{m}$. Then, by Lemma 5.1, there exists a positive constant $C$ independent of $t$ such that

$$
\left|\left(\frac{\mathbf{k}(t)}{\widehat{\mathbf{k}}(t)}\right)\right|^{2} \leq C \quad \text { for all } t \in\left(t_{0}-\varepsilon, t_{m}\right) .
$$

Therefore, it follows from an argument similar to that in [10, Section 4 of Ch. 1] that the limit value $\lim _{t \rightarrow t_{m}-0}\left(\begin{array}{c}\mathbf{k}(t) \\ \widehat{\mathbf{k}}(t)\end{array}\right) \in \mathbb{R}^{2(n-1)}$ exists and ${ }^{t}(\mathbf{k}, \widehat{\mathbf{k}})$ can be continued to the right of $t_{m}$. This contradicts the assumption. Hence the local solution ${ }^{t}(\mathbf{k}, \widehat{\mathbf{k}})$ can be continued to $\left(t_{0}-\varepsilon, \infty\right)$.

Similarly, it follows that the local solution ${ }^{t}(\mathbf{k}, \widehat{\mathbf{k}})$ can be continued to $\left(-\infty, t_{0}+\varepsilon\right)$. Consequently, ${ }^{t}(\mathbf{k}, \widehat{\mathbf{k}})$ can be continued to the whole of $\mathbb{R}$. Hence there exists a global solution $\mathbf{k}: \mathbb{R} \rightarrow \mathbb{R}^{n-1}$ of the initial-value problem (5.3) and (5.4). The proof of the claim is complete.

\section{References}

[1] S. S. Antman, 'Ordinary differential equations of nonlinear elasticity. II. Existence and regularity theory for conservative boundary value problem', Arch. Ration. Mech. Anal. 61(4) (1976), 353-393.

[2] S. S. Antman, Nonlinear Problems of Elasticity, Applied Mathematical Sciences, 107 (Springer, New York, 1995).

[3] S. S. Antman and K. B. Jordan, 'Qualitative aspects of the spatial deformation of non-linearly elastic rods', Proc. Roy. Soc. Edinburgh Sect. A 73 (1975), 85-105.

[4] J. Arroyo, O. J. Garay and J. J. Mencía, 'Closed generalized elastic curves in $S^{2}(1)$ ', J. Geom. Phys. 48(2-3) (2003), 339-353.

[5] J. Arroyo, O. J. Garay and J. J. Mencía, 'Extremals of curvature energy actions on spherical closed curves', J. Geom. Phys. 51(1) (2004), 101-125.

[6] J. Arroyo, O. J. Garay and J. J. Mencía, 'Quadratic curvature energies in the 2-sphere', Bull. Aust. Math. Soc. 81(3) (2010), 496-506.

[7] M. Barros, O. J. Garay and D. A. Singer, 'Elasticae with constant slant in the complex projective plane and new examples of Willmore tori in five spheres', Tohoku Math. J. (2) 51(2) (1999), 177-192.

[8] R. L. Bishop, 'There is more than one way to frame a curve', Amer. Math. Monthly 82 (1975), 246-251.

[9] R. Bryant and P. A. Griffiths, 'Reduction for constrained variational problems and $\int \frac{1}{2} k^{2} d s^{\prime}$, Amer. J. Math. 108(3) (1986), 525-570.

[10] E. A. Coddington and N. Levinson, Theory of Ordinary Differential Equations (McGraw-Hill, New York, 1955).

[11] B. D. Coleman, E. H. Dill, M. Lembo, Z. Lu and I. Tobias, 'On the dynamics of rods in the theory of Kirchhoff and Clebsch', Arch. Ration. Mech. Anal. 121(4) (1992), 339-359.

[12] O. J. Garay, 'Extremals of the generalized Euler-Bernoulli energy and applications', J. Geom. Symmetry Phys. 12 (2008), 27-61.

[13] P. A. Griffiths, Exterior Differential Systems and the Calculus of Variations, Progress in Mathematics, 25 (Birkhäuser, Boston, 1983).

[14] J. Inoguchi and J.-E. Lee, 'Biminimal curves in 2-dimensional space forms', Commun. Korean Math. Soc., to appear. 
[15] T. A. Ivey and D. A. Singer, 'Knot types, homotopies and stability of closed elastic rods', Proc. Lond. Math. Soc. (3) 79(2) (1999), 429-450.

[16] V. Jurdjevic, 'Non-Euclidean elastica', Amer. J. Math. 117(1) (1995), 93-124.

[17] V. Jurdjevic, Geometric Control Theory, Cambridge Studies in Advanced Mathematics, 52 (Cambridge University Press, Cambridge, 1997).

[18] V. Jurdjevic, 'Integrable Hamiltonian systems on complex Lie groups', Mem. Amer. Math. Soc. 178(838) (2005).

[19] S. Kawakubo, 'Kirchhoff elastic rods in a Riemannian manifold', Tohoku Math. J. (2) 54(2) (2002), 179-193.

[20] S. Kawakubo, 'Kirchhoff elastic rods in the three-sphere', Tohoku Math. J. (2) 56(2) (2004), 205-235.

[21] S. Kawakubo, 'Kirchhoff elastic rods in three-dimensional space forms', J. Math. Soc. Japan 60(2) (2008), 551-582.

[22] S. Kawakubo, 'First integrals and global solutions of the equation for Kirchhoff elastic rods', Fukuoka Univ. Sci. Rep. 41(2) (2011), 147-153.

[23] S. Kawakubo, 'Kirchhoff elastic rods in higher-dimensional space forms', Proc. Japan Acad. Ser. A Math. Sci. 87(1) (2011), 5-9.

[24] S. Kehrbaum and J. H. Maddocks, 'Elastic rods, rigid bodies, quaternions and the last quadrature', Philos. Trans. R. Soc. Lond. Ser. A 355(1732) (1997), 2117-2136.

[25] S. Kobayashi and K. Nomizu, Foundations of Differential Geometry. Vol. I. Wiley Classics Library (Wiley, New York, 1996), Reprint of the 1963 original.

[26] N. Koiso, 'Elasticae in a Riemannian submanifold', Osaka J. Math. 29(3) (1992), 539-543.

[27] N. Koiso, 'Convergence towards an elastica', in: Geometry and Global Analysis (Sendai, 1993) (Tohoku Univ., Sendai, 1993), pp. 249-254.

[28] N. Koiso, 'Convergence towards an elastica in a Riemannian manifold', Osaka J. Math. 37(2) (2000), 467-487.

[29] J. Langer and D. A. Singer, 'The total squared curvature of closed curves', J. Differential Geom. 20(1) (1984), 1-22.

[30] J. Langer and D. A. Singer, 'Liouville integrability of geometric variational problems', Comment. Math. Helv. 69(2) (1994), 272-280.

[31] J. Langer and D. A. Singer, 'Lagrangian aspects of the Kirchhoff elastic rod', SIAM Rev. 38(4) (1996), 605-618.

[32] A. Linnér, 'Curve-straightening in closed Euclidean submanifolds', Comm. Math. Phys. 138(1) (1991), 33-49.

[33] E. Loubeau and S. Montaldo, 'Biminimal immersions', Proc. Edinb. Math. Soc. (2) 51(2) (2008), 421-437.

[34] A. E. H. Love, A Treatise on the Mathematical Theory of Elasticity, 4th edn (Dover Publications, New York, 1944).

[35] M. Nizette and A. Goriely, 'Towards a classification of Euler-Kirchhoff filaments', J. Math. Phys. 40(6) (1999), 2830-2866.

[36] U. Pinkall, 'Hopf tori in $S^{3}$ ', Invent. Math. 81(2) (1985), 379-386.

[37] T. Popiel and L. Noakes, 'Elastica in SO(3)', J. Aust. Math. Soc. 83(1) (2007), 105-124.

[38] Y. Shi and J. Hearst, 'The Kirchhoff elastic rod, the nonlinear Schrödinger equation, and DNA supercoiling', J. Chem. Phys. 101 (1994), 5186-5200.

[39] H. Tsuru, 'Equilibrium shapes and vibrations of thin elastic rod', J. Phys. Soc. Japan 56(7) (1987), $2309-2324$.

\section{SATOSHI KAWAKUBO, Department of Applied Mathematics, Fukuoka University, Fukuoka 814-0180, Japan \\ e-mail: kawakubo@math.sci.fukuoka-u.ac.jp}

